\title{
Prospective US investigational device exemption trial of a sutureless aortic bioprosthesis: One-year outcomes
}

\author{
Rakesh M. Suri, MD, DPhil, ${ }^{\mathrm{a}}$ Hoda Javadikasgari, MD, ${ }^{\mathrm{a}}$ David A. Heimansohn, MD, ${ }^{\mathrm{b}}$ \\ Neil J. Weissman, MD, ${ }^{\mathrm{c}}$ Gorav Ailawadi, MD, ${ }^{\mathrm{d}}$ Niv Ad, MD, ${ }^{\mathrm{e}}$ Gabriel S. Aldea, MD, \\ Vinod H. Thourani, MD, ${ }^{\mathrm{g}}$ Wilson Y. Szeto, MD,${ }^{\mathrm{h}}$ Robert E. Michler, MD, ${ }^{\mathrm{i}}$ Hector I. Michelena, MD, ${ }^{\mathrm{j}}$ \\ Reza Dabir, MD, ${ }^{\mathrm{k}}$ Gregory P. Fontana, MD, ${ }^{\mathrm{l}}$ William F. Kessler, MD, ${ }^{\mathrm{m}}$ Michael G. Moront, MD, ${ }^{\mathrm{n}}$ \\ Louis A. Brunsting III, MD, ${ }^{\circ}$ Bartley P. Griffith, MD, ${ }^{\mathrm{p}}$ Alvaro Montoya, MD, ${ }^{\mathrm{q}}$ \\ Sreekumar Subramanian, MD, ${ }^{\mathrm{r}}$ Mark A. Mostovych, MD, ${ }^{\mathrm{s}}$ and Eric E. Roselli, MD ${ }^{\mathrm{a}}$
}

\section{ABSTRACT}

Objectives: We performed a prospective, single-arm clinical trial approved under a Food and Drug Administration Investigational Device Exemption to assess safety and efficacy of Perceval, a sutureless bovine pericardial aortic valve representing the initial US experience.

Methods: From June 2013 to January 2015, 300 patients (mean age $76.7 \pm 7.7$ years, $54.3 \%$ men, $37.3 \% \geq 80$ years, median Society of Thoracic Surgeons Predicted Risk of Mortality $2.8 \%$ ), underwent Perceval valve implantation at 18 centers across the United States. Twenty patients $(6.7 \%)$ had a bicuspid aortic valve and $5(1.7 \%)$ patients had previous aortic valve replacement. A minimally invasive approach was used in 80 (26.7\%) and concomitant procedures were performed in $113(37.8 \%)$ patients.

Results: Two hundred eighty-nine patients (96.3\%) were successfully implanted. Operative mortality $(\leq 30$ days) was $1.3 \%(n=4)$ (observed to expected ratio of $0.40)$. One-year results included all-cause mortality in $5.2 \%(\mathrm{n}=15)$, stroke in $1 \%(\mathrm{n}=3)$, and endocarditis in $1.7 \%(\mathrm{n}=5)$. New permanent periprocedural pacemaker rate was $10.7 \%(\mathrm{n}=30 / 281) ; 2.5 \%(\mathrm{n}=7 / 281)$ resulted from third-degree atrioventricular block. One-year valve-related reoperation was $2.1 \%(n=6)$. At 1-year follow-up, $98 \%$ of patients were in New York Heart Association class I/II, left ventricular mass index decreased from $103.5 \pm 30.1 \mathrm{~g} / \mathrm{m}^{2}$ at discharge to $95.8 \pm 27.1 \mathrm{~g} / \mathrm{m}^{2}(P=.001)$, and $3(1.3 \%)$ moderate paravalvular leaks were identified. Health-related quality of life score increased from $62.7 \pm 21.8$ before surgery to $85.5 \pm 17.8$ at 1 year $(P<.001)$.

Conclusions: These results confirm the safety and effectiveness of the Perceval sutureless aortic valve replacement in study patients with lower mortality than

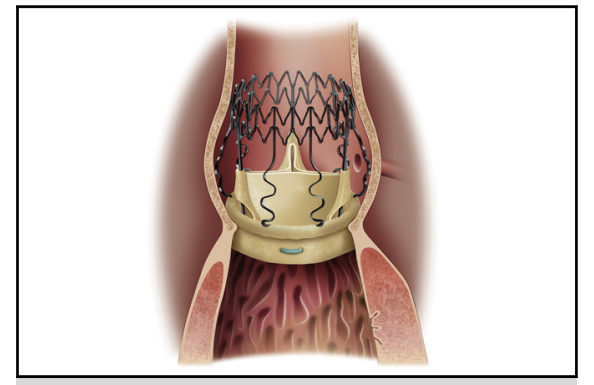

Perceval Sutureless aortic valve

\section{Central Message}

The results of the initial US experience for Perceval in a prospective, single-arm clinical trial confirm safety and efficacy outcomes both in isolated and combined procedures regardless of surgical approach.

\section{Perspective}

There is an extensive medical literature for Perceval from centers outside of the United States; this initial US experience further confirms sutureless aortic valve replacement is a safe and effective therapy for patients with aortic valve stenosis. The device may be used in isolated and combined procedures via open or minimally invasive approaches.

See Commentary on page 1783.

\begin{abstract}
From the a Department of Thoracic and Cardiovascular Surgery, Heart and Vascular Institute, Cleveland Clinic Abu Dhabi and Cleveland Clinic Foundation, Cleveland, Ohio; ' Department of Cardiothoracic Surgery, St Vincent Heart Center of Indiana, Indianapolis, Ind; 'Cardiovascular Core Laboratories, MedStar Health Research Institute, Washington, DC; ${ }^{\mathrm{d}}$ Section of Adult Cardiac Surgery, Advanced

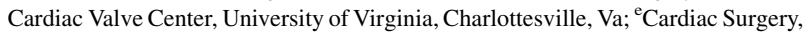
The Inova Heart and Vascular Institute, West Virginia University Medical Center, Morgantown, WV; ${ }^{\mathrm{f}}$ Division of Cardiothoracic Surgery, University of Washington Medical Center, Seattle, Wash; ${ }^{g}$ Cardiothoracic Surgery, Emory Midtown Hospital, Atlanta, Ga; ${ }^{\mathrm{h}}$ Division of Cardiovascular Surgery, Department of Surgery, University of Pennsylvania Medical Center, Philadelphia, Pa; ${ }^{\mathrm{i}}$ Cardiothoracic Surgery and Surgery, Montefiore-Einstein Heart Center, New York, NY; ${ }^{j}$ Department of Cardiovascular Medicine, Mayo Clinic, Rochester, Minn; ${ }^{k}$ Cardiovascular and

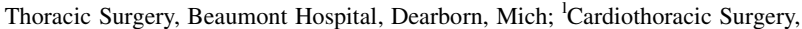
Cardiovascular Institute, Los Robles Hospital and Medical Center, Thousand Oaks CA Hospital Corporation of America, Nashville, Tenn; ${ }^{\mathrm{m}}$ Cardiothoracic and Vascular Surgeons, Heart Hospital, Austin, Tex; ${ }^{\mathrm{n} C a r d i o t h o r a c i c ~ S u r g e r y, ~}$ The Toledo Hospital, Toledo, Ohio; ${ }^{\circ}$ Heart and Lung Surgeons of East Alabama, East Alabama Medical Center (EAMC), Opelika, Ala; ${ }^{\mathrm{P}}$ Division of Cardiac
\end{abstract}

Surgery, University of Maryland, Baltimore, Md; ${ }^{9}$ Cardiothoracic Surgery, Baptist Health, South Florida, Fla; ${ }^{\mathrm{r}}$ Department of Cardiac Surgery, TriStar Centennial Medical Center, Nashville, Tenn; and ${ }^{\mathrm{S}}$ Cardiac and Vascular Surgery, St Vincent's Medical Center, Jacksonville, Fla

This trial (Clinical trial registry number: NCT01810679) was a medical investigational device study conducted under the authority of the Food and Drug Administration and supported by the Sponsor, LivaNova PLC, London, United Kingdom. Read at the 97th Annual Meeting of The American Association for Thoracic Surgery, Boston, Massachusetts, April 29-May 3, 2017.

Received for publication May 20, 2017; revisions received Aug 5, 2018; accepted for publication Aug 14, 2018; available ahead of print Dec 12, 2018.

Address for reprints: Rakesh M. Suri, MD, DPhil, Department of Thoracic and Cardiovascular Surgery, Heart and Vascular Institute, Cleveland Clinic Abu Dhabi and Cleveland Clinic Foundation, 9500 Euclid Ave, Cleveland, OH 44195 (E-mail: surir@ccf.org).

0022-5223/\$36.00

Copyright $(\subset) 2018$ by The American Association for Thoracic Surgery https://doi.org/10.1016/j.jtcvs.2018.08.121 


\section{Abbreviations and Acronyms \\ AVR = aortic valve replacement \\ BAV = bicuspid aortic valve \\ CAVALIER $=$ Perceval $\mathrm{S}$ valve clinical trial for extended CE mark \\ $\mathrm{EOA}=$ effective orifice area \\ HR-QoL = health-related quality of life \\ ICU = intensive care unit \\ IDE = Investigational Device Exemption \\ KCCQ = Kansas City Cardiomyopathy Questionnaire \\ $\mathrm{LV} \quad=$ left ventricle \\ NYHA $=$ New York Heart Association \\ PMI $=$ pacemaker insertion \\ PVL $\quad$ paravalvular leak \\ STS-PROM $=$ Society of Thoracic Surgeons Predicted Risk of Mortality}

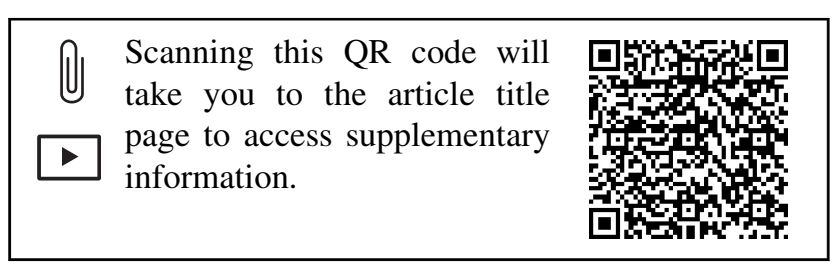

expected from a risk prediction model. Persistent hemodynamic benefit and improvement in quality of life at 1 year support the importance of this device in the management of aortic valve disease. ( $\mathrm{J}$ Thorac Cardiovasc Surg 2019;157:1773-82)

Aortic valve replacement (AVR) is the procedure of choice for patients with severe symptomatic aortic stenosis. ${ }^{1}$ These patients often have multiple age-related comorbidities that may increase the risk of conventional surgical AVR. ${ }^{2-4}$ Sutureless AVR has emerged as a promising technique with good hemodynamic properties and is a viable option for patients with high surgical risk. ${ }^{5}$ The concept of sutureless bioprosthetic aortic valves combines the advantage of aortic annular debridement and the possibility of performing concomitant procedures with shorter aortic crossclamp and procedural times compared with standard surgical AVR. The Perceval Sutureless Aortic Valve Prosthesis (LivaNova, London, United Kingdom) was introduced in 2007. Three European trials have demonstrated its safety and efficacy in intermediate- and high-risk patients with severe symptomatic aortic stenosis. ${ }^{6-10}$ Shorter procedure times, favorable hemodynamic performance, and facilitation of minimally invasive surgical approaches have been documented in this experience.

The first North-American experience occurred in Canada and confirmed excellent short-term hemodynamic performance and clinical outcomes. ${ }^{11}$ The current report documents the initial US experience, which was a prospective trial performed under a Food and Drug Administration Investigational Device Exemption (IDE).

\section{METHODS}

\section{Study Design}

The Perceval IDE trial was designed as a multicenter, prospective, nonrandomized, single-arm clinical trial (NCT01810679; www.clinicaltrials. gov) to evaluate the safety and efficacy of the Perceval valve (LivaNova). The institutional review board at each participating institution approved the study, and all patients provided written informed consent. Inclusion and exclusion criteria are listed in Table E1 and the investigational plan is provided as an Online Data Supplement.

An independent Clinical Events Committee adjudicated all reported instances of death, explantation, valve dysfunction, and adverse events as requested by the investigators or the independently appointed Data Safety Monitoring Board during their periodic review. The investigators had full authority to publish the data without restriction from the sponsoring company.

\section{Surgical Technique}

The Perceval sutureless valve is a new-generation bioprosthetic aortic valve composed of glutaraldehyde-fixed bovine pericardial leaflets treated with homocysteic acid to mitigate calcification within a stent made of a super-elastic nickel-titanium alloy (Nitinol) covered by Carbofilm (LivaNova) (Figure 1). The features and implantation regimen have been described previously. ${ }^{12}$ To summarize, the valve is collapsed with a dedicated accessory device and is deployed using a delivery system with stepwise release of the valve inflow and outflow, followed by postimplantation dilation performed with a specifically designed low-pressure balloon catheter at 4 atmospheres pressure. The valve is available in 4 sizes: $\mathrm{S}, \mathrm{M}, \mathrm{L}$, and XL, corresponding to annulus sizes from 19 to $27 \mathrm{~mm}$.

In the present study, the valve was implanted via full median sternotomy, partial upper sternotomy, or right anterolateral thoracotomy, according to individual surgeon preference. Postoperative anticoagulation was left to the discretion of individual investigators and was performed according to patients' clinical status and individual center standard practice.

\section{Follow-up}

Patients were evaluated at the following time points: preoperatively, at hospital discharge/within 30 days, postoperatively between 3 and 6 months, and at 12 months. All patients underwent clinical evaluation, laboratory testing, and transthoracic echocardiography at each followup visit. Intraoperative transesophageal echocardiography was used in all patients.

\section{Echocardiography}

Aortic geometry and diameters were determined from 2-dimensional parasternal long-axis views. Subaortic and aortic Doppler flow velocities were recorded from the apical 3-chamber or 5-chamber view using pulse-wave and continuous-wave Doppler, respectively. Peak and mean aortic valve pressure gradients and effective orifice area (EOA) were ascertained; peak and mean aortic valve pressure gradients were calculated from velocity measurements using a modified Bernoulli equation. ${ }^{13}$ All echocardiographic examinations were blinded for patient status and analyzed by an independent echocardiography core laboratory (MedStar Health Research Institute, Washington, DC). 


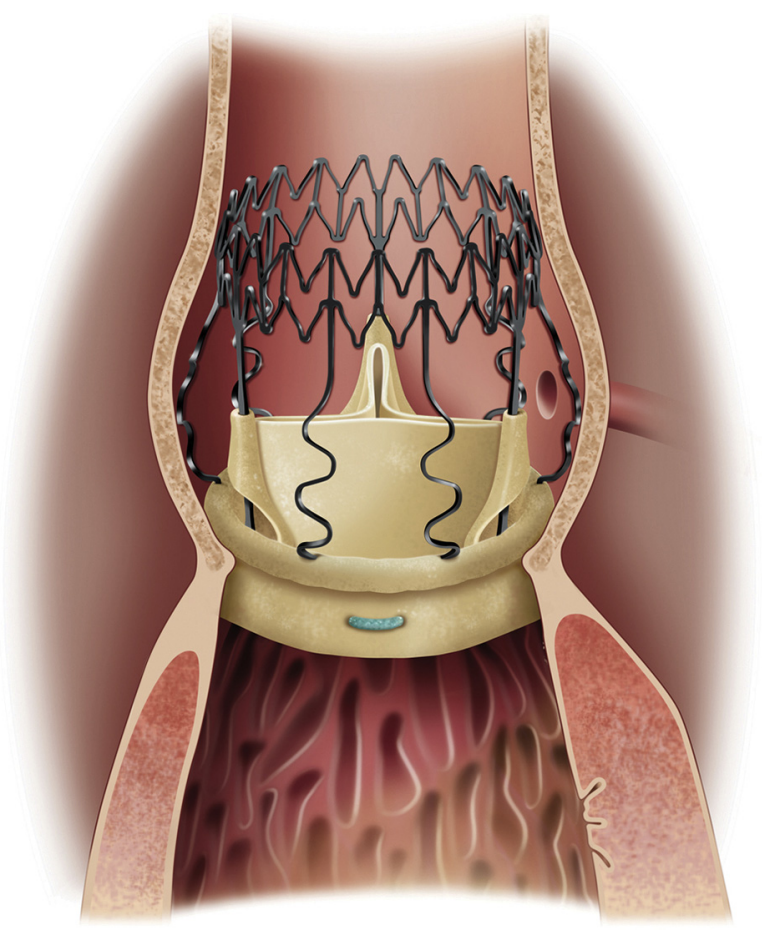

FIGURE 1. Perceval sutureless aortic valve.

\section{Quality of Life}

The Kansas City Cardiomyopathy Questionnaire (KCCQ) was used to assess disease-specific health status, and its overall summary score to provide health-related quality of life (HR-QoL) measures for each patient. The KCCQ is a 23-item questionnaire designed and validated for self-reported health status in patients with heart failure ${ }^{14}$ The conceptual domains of the KCCQ include symptoms, physical limitation, social limitation, selfefficacy, and quality of life. These scales, as well as an overall summary scale, are scored from 0 to 100 , with greater scores indicating fewer symptoms and better HR-QoL.

\section{Study Objectives and End Points}

The Perceval US IDE Trial was designed to assess device safety and effectiveness. Safety of the Perceval valve was evaluated based on the following adverse events: valve thrombosis, thromboembolism, perivalvular leak (all and major), hemorrhage (all and major), and endocarditis. The primary composite effectiveness endpoints included implant success (defined as leaving the operating room with the originally implanted Perceval valve in place); absence of moderate-to-severe paravalvular leak (PVL); freedom from structural dysfunction; and absence of migration. Secondary effectiveness endpoint was implant success in all patients with an attempted implant $(n=300)$. Video 1 provides a summary of the main features of the present study.

\section{Data Analysis}

Categorical variables are reported as absolute and relative frequencies. For continuous data, means and standard deviations were calculated. Where clinically relevant, statistical comparisons between groups were performed using the Fisher exact test for categorical data and Student $t$ test for continuous data.

Cumulative survival and freedom from events were estimated using the Kaplan-Meier method, with $95 \%$ confidence intervals until a cut-off follow-up of 365 days. The degree of uncertainty in each actuarial analysis

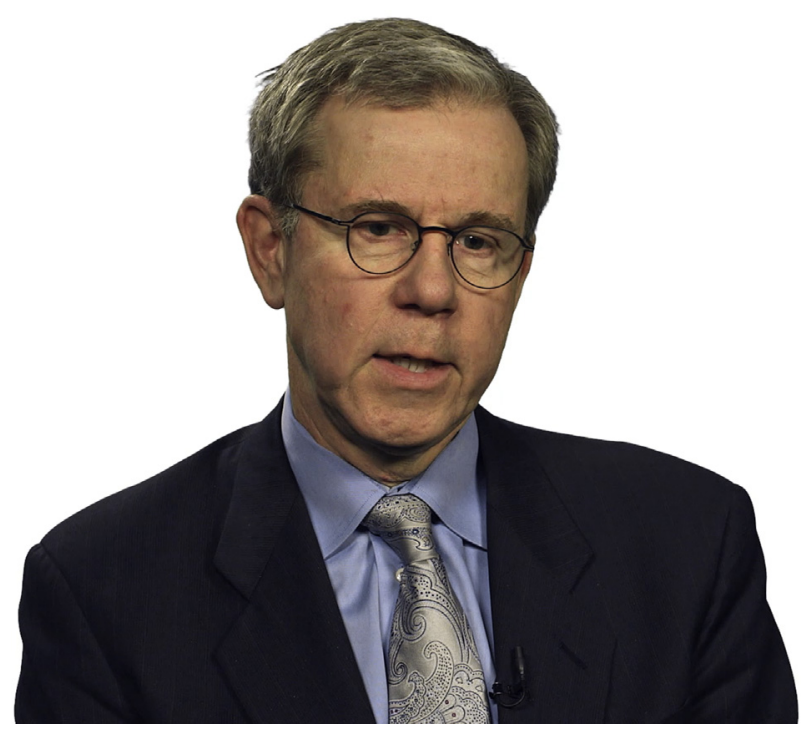

VIDEO 1. This narrated video describes the main features of the Investigational Device Exemption trial as well as the main advantages related to the use of the Perceval valve, demonstrating the unique features of this sutureless aortic valve. Video available at: https://www.jtcvs.org/article/ S0022-5223(18)32824-1/fulltext.

is expressed with $95 \%$ confidence limits. The $95 \%$ confidence interval bounds for the cumulative freedom were calculated according to the method proposed by Greenwood. ${ }^{15}$

Site-reported adverse events at 30 days (0-30 days), at 3 to 6 months, and at 1-year rates were reported as number of events divided by the total number of patients in which the implant was attempted. ${ }^{16}$ Statistical analyses were performed using SAS software (version 9.4; SAS Institute Inc, Cary, NC). For all tests, a $P$ of .05 or less (2-sided) was considered as statistically significant, unless otherwise specified.

\section{RESULTS}

\section{Patients Characteristics}

From June 2013 to January 2015, a total of 356 patients were enrolled from 18 US centers. Fifty-six $(15.7 \%)$ patients were excluded according to predetermined criteria (Figure E1) because of patient anatomic reasons $(n=14$, $25 \%$ ), aortic annulus size exceeding the largest available prosthesis size $(\mathrm{n}=14,25 \%)$, patient/surgeon preference $(\mathrm{n}=16,29.1 \%)$, other reasons $(\mathrm{n}=9)$, and patient death before surgery $(\mathrm{n}=3,5.4 \%)$. Anatomic reasons for study exclusion included patients with a ratio of the diameters of the sino-tubular junction to the aortic annulus greater than $1.3(n=4)$, aortic root aneurysm $(n=4)$, bicuspid valve $(n=1)$, enlarged annulus $(n=1)$, unspecified anatomic reasons $(\mathrm{n}=2)$, heavily calcified aorta $(\mathrm{n}=1)$, and concentric left ventricular hypertrophy $(\mathrm{n}=1)$.

A Perceval valve was successfully implanted in 289 of 300 patients for a success rate of $96.3 \%$. Eleven patients had an unsuccessful implantation attempt: 6 patients had the valve removed due to PVL $(\mathrm{n}=4)$, or device dislodgement $(\mathrm{n}=2)$, with the subsequent decision to proceed with standard surgical valve replacement; 2 patients could not be 
sized appropriately due to bicuspid valve disease $(n=1)$ or an annulus outside the study parameters $(\mathrm{n}=1) ; 2$ patients suffered annular bleeding probably related to annular debridement and required standard AVR; 1 patient suffered a limited aortic dissection related to aortic calcification and required root replacement.

Mean age was $76.7 \pm 7.7$ years, and $37.3 \%(n=112)$ of patients were octogenarians. The median Society of Thoracic Surgeons Predicted Risk of Mortality (STSPROM) was 2.8 (interquartile range of 1.9-4.2). The majority of patients underwent full sternotomy $(n=220 ; 73.3 \%)$, and $43.3 \%$ received a size $\mathrm{L}(\mathrm{n}=130)$. Coronary artery bypass grafting was the most common concomitant procedure $(\mathrm{n}=89 ; 29.7 \%)$. Overall mean aortic crossclamp time was $53.2 \pm 22.8$ minutes, $44.5 \pm 13.8$ minutes for isolated AVR and $71 \pm 21.5$ minutes for AVR with concomitant procedures. Preoperative and operative patient data are shown in Tables 1 and 2, for the overall patient cohort and for patients with bicuspid aortic valve (BAV). Mean intensive care unit (ICU) and hospital length of stay were $3.1 \pm 3.0$ days and $8.0 \pm 4.7$ days, respectively. Mean ICU and hospital lengths of stay were longer for the initial 20 patients included in each center compared with patients managed later in the experience (ICU: $3.4 \pm 3.4$ vs $2.4 \pm 1.8$ days; length of stay: $8.4 \pm 4.9$ vs $7.1 \pm 4.2$ ).

At discharge, the majority of patients $(56.1 \% ; 161 / 287)$ were on single antiplatelet therapy (acetylsalicylic acid, clopidogrel, other) or in combination with oral anticoagulants (warfarin) $(24.7 \%$; 71/287). Only 1 patient was maintained on warfarin alone. Dual antiplatelet therapy was given to $17.4 \%(50 / 287)$ of patients. At 1 year, $63.3 \%$ of patients $(155 / 245)$ were treated with single antiplatelet therapy, or in combination with oral anticoagulants $(14.3 \%$; 35/ 245); the remaining patients were on dual antiplatelet therapy $(10.2 \% ; 25 / 245)$ or on warfarin alone $(5.7 \% ; 14 / 245)$.

\section{Periprocedural Outcomes}

Four patients died after Perceval implantation before discharge, for an operative mortality of $1.3 \%$. Three patients $(0.7 \%)$ died due to cardiovascular causes (bleeding from aortic tear 5 days after procedure, cardiopulmonary arrest 3 days postsurgery, and 1 procedure-related death 3 days postsurgery due to aortic dissection in a patient who experienced a failure to implant of the Perceval valve and underwent a traditional valve implant) and $1(0.3 \%)$ due to noncardiovascular cause (ischemic hepatitis 3 days after surgery) (Table 3). A stroke occurred in 2 patients $(0.7 \%)$, and a postoperative pacemaker implantation (PMI) was required in $10.7 \%$ (30/281); 2.5\% (7/281) of these resulted from third-degree atrioventricular block.

\section{Time-Related Outcomes}

Mortality. A total of 11 late deaths occurred between the 30 -day and 1-year time points including $6(2.1 \%)$ due to
TABLE 1. Baseline characteristics: implant attempted patients $(\mathbf{N}=\mathbf{3 0 0})$

\begin{tabular}{|c|c|c|}
\hline \multirow{2}{*}{$\begin{array}{r}\text { Variables } \\
\text { Demographic }\end{array}$} & \multicolumn{2}{|c|}{ Number $(\%)$ or mean \pm SD } \\
\hline & All $(\mathrm{N}=300)$ & $\mathrm{BAV}(\mathrm{N}=20)$ \\
\hline Age, y & $76.7 \pm 7.7$ & $67.9 \pm 7.1$ \\
\hline$<80$ & $188(62.7)$ & $19(95)$ \\
\hline$\geq 80$ & $112(37.3)$ & $1(5)$ \\
\hline BSA, $m^{2}$ & $1.9 \pm 0.3$ & $1.9 \pm 0.2$ \\
\hline Sex, male & $163(54.3)$ & $10(50)$ \\
\hline \multicolumn{3}{|l|}{ Previous cardiac procedures* } \\
\hline Cardiac surgery & $40(13.3)$ & $1(5)$ \\
\hline AVR & $5(1.7)$ & $1(5)$ \\
\hline Aortic valve repair & $1(0.3)$ & - \\
\hline Aortic aneurysm repair & $1(0.3)$ & - \\
\hline CABG & $13(4.3)$ & - \\
\hline Others & $10(3.3)$ & - \\
\hline $\begin{array}{l}\text { Cardiac interventional } \\
\text { procedures }\end{array}$ & $125(41.7)$ & $4(20)$ \\
\hline $\mathrm{PCI} / \mathrm{PCI}+$ stent & $57(19.0)$ & $1(5)$ \\
\hline Pacemaker/defibrillator & $19(6.3)$ & - \\
\hline Other & $49(16.3)$ & $3(15)$ \\
\hline \multicolumn{3}{|l|}{ Risk score } \\
\hline Mean STS score & $3.3 \pm 2.3$ & $2.0 \pm 1.5$ \\
\hline Median STS score [Q1; Q3] & $2.8[1.9 ; 4.2]$ & $1.4[1.0 ; 3.0]$ \\
\hline \multicolumn{3}{|l|}{ NYHA class } \\
\hline I & $22(7.5)$ & $3(15)$ \\
\hline II & $146(49.5)$ & $13(65)$ \\
\hline III & $118(40.0)$ & $4(20)$ \\
\hline IV & $9(3.1)$ & $0(0)$ \\
\hline Missing & 5 & - \\
\hline \multicolumn{3}{|l|}{ Preoperative comorbidities } \\
\hline Dyslipidemia & $256(85.3)$ & $17(85)$ \\
\hline Hypertension & $246(82.0)$ & $13(65)$ \\
\hline Coronary artery disease & $179(59.7)$ & $7(35)$ \\
\hline Angina & $65(21.7)$ & $3(15)$ \\
\hline Tobacco use & $166(55.3)$ & $6(30)$ \\
\hline Diabetes & $103(34.3)$ & $8(40)$ \\
\hline Arrhythmia & $95(31.7)$ & $5(25)$ \\
\hline Chronic lung disease & $72(24.0)$ & $3(15)$ \\
\hline Cerebrovascular disease & $39(13.0)$ & $1(5)$ \\
\hline Peripheral vascular disease & $34(11.3)$ & $3(15)$ \\
\hline Pulmonary hypertension & $32(10.7)$ & $3(15)$ \\
\hline Myocardial infarction $(>30 \mathrm{~d})$ & $30(10.0)$ & $0(0)$ \\
\hline $\begin{array}{l}\text { Pre-existing conduction } \\
\text { abnormality }\end{array}$ & $27(9.0)$ & $1(5)$ \\
\hline Renal failure & $15(5.0)$ & $0(0)$ \\
\hline Neurologic dysfunction & $11(3.7)$ & $1(5)$ \\
\hline Immunosuppressive treatment & $8(2.7)$ & $1(5)$ \\
\hline \multicolumn{3}{|l|}{ Hemodynamic characteristics } \\
\hline $\begin{array}{l}\text { Left ventricular ejection } \\
\text { fraction }\end{array}$ & $60.5 \pm 9.9$ & $64.7 \pm 7.5$ \\
\hline Mean aortic gradient, $\mathrm{mm} \mathrm{Hg}$ & $45.4 \pm 14.7$ & $50.4 \pm 10.9$ \\
\hline Effective orifice area, $\mathrm{cm}^{2}$ & $0.8 \pm 0.2$ & $0.7 \pm 0.2$ \\
\hline
\end{tabular}

$S D$, Standard deviation; $B A V$, bicuspid aortic valve; $B S A$, body surface area; $A V R$, aortic valve replacement; $C A B G$, coronary artery bypass graft; $P C I$, percutaneous coronary intervention; STS, Society of Thoracic Surgeons; $Q 1 ; Q 3$, interquartile range; NYHA, New York Heart Association. *Subjects may have more than one previous cardiac procedures. 
TABLE 2. Operative characteristics: implanted attempted patients $(\mathbf{N}=\mathbf{3 0 0})$

\begin{tabular}{|c|c|c|}
\hline & \multicolumn{2}{|c|}{ Number $(\%)$ or mean \pm SD } \\
\hline & All $(\mathbf{N}=300)$ & BAV $(\mathbf{N}=\mathbf{2 0})$ \\
\hline \multicolumn{3}{|l|}{ Surgical approach } \\
\hline Full sternotomy & $220(73.3)$ & $13(65.0)$ \\
\hline Minimally invasive & $80(26.7)$ & $7(35.0)$ \\
\hline Mini-sternotomy & $67(22.3)$ & $4(20.0)$ \\
\hline $\begin{array}{l}\text { Right anterior } \\
\text { thoracotomy }\end{array}$ & $3(1.0)$ & $3(15.0)$ \\
\hline $\begin{array}{l}\text { Minimally invasive } \\
\text { unspecified }\end{array}$ & $10(3.3)$ & - \\
\hline \multicolumn{3}{|l|}{ Aortic valve diagnosis } \\
\hline Stenosis & $169(56.3)$ & $12(60.0)$ \\
\hline Stenoregurgitation & $126(42.0)$ & $8(40.0)$ \\
\hline $\begin{array}{l}\text { Previous prosthetic } \\
\text { structural valve } \\
\text { deterioration }\end{array}$ & $5(1.7)$ & - \\
\hline \multicolumn{3}{|l|}{ Aortic valve etiology } \\
\hline Calcification & $291(97.0)$ & $19(95.0)$ \\
\hline Congenital bicuspid valve & $20(6.7)$ & - \\
\hline Prosthetic valve in place & $5(1.7)$ & - \\
\hline Other etiology* & $7(2.3)$ & $1(5.0)$ \\
\hline \multicolumn{3}{|l|}{ Valve size } \\
\hline$S$ & $21(7.0)$ & $3(15.0)$ \\
\hline M & $101(33.7)$ & $6(30.0)$ \\
\hline $\mathrm{L}$ & $130(43.3)$ & $9(45.0)$ \\
\hline XL & $48(16.0)$ & $2(10.0)$ \\
\hline \multicolumn{3}{|l|}{ Procedure $\dagger$} \\
\hline Isolated AVR & $186(62.2)$ & 15 (78.9) \\
\hline Concomitant procedures $\ddagger$ & $113(37.8)$ & $4(21.1)$ \\
\hline CABG & 89 (29.7) & $4(21.1)$ \\
\hline LAA ligation/exclusion & $20(6.7)$ & - \\
\hline Septal myectomy & $3(1.0)$ & - \\
\hline Maze/ablation & $1(0.3)$ & - \\
\hline Other procedures $\S$ & $15(5.0)$ & - \\
\hline \multicolumn{3}{|l|}{ Surgical time } \\
\hline Perceval implant time, min & $22.1 \pm 20.8$ & $25.7 \pm 40.1$ \\
\hline Crossclamp time, min & $53.2 \pm 22.8$ & $52.3 \pm 19.6$ \\
\hline $\begin{array}{l}\text { Cardiopulmonary bypass } \\
\text { time, min }\end{array}$ & $75.0 \pm 30.9$ & $70.2 \pm 27.8$ \\
\hline
\end{tabular}

$S D$, Standard deviation; $B A V$, bicuspid aortic valve; $A V R$, aortic valve replacement; $C A B G$, coronary artery bypass graft; $L A A$, left atrial appendage. *Subject may have more than 1 etiology. Other etiology includes congenital anomaly, rheumatic heart disease, and collagen vascular disease. †Missing information for 1 patient in the bicuspid cohort. ‡Subject may have more than 1 concomitant procedure. §Other procedures include annular repair (2), endarterectomy of ascending aorta, repair of right ventricular laceration, proximal aortic endarterectomy, lung resection (2), intra-aortic balloon pump implant, other noncardiac procedures.

cardiovascular causes: cardiorespiratory failure $(n=3)$, acute myocardial ischemia $(\mathrm{n}=2)$, and endocarditis $(\mathrm{n}=1)$. Five late deaths were due to noncardiovascular events (Table 3). All-cause mortality at 1 year was 3.8\% $(\mathrm{n}=7 / 182)$ for isolated AVR and 7.5\% $(\mathrm{n}=8 / 107)$ for AVR with concomitant cardiac procedures. Survival was $96.18 \%$ at 3 to 6 months and $94.74 \%$ at 12 months (Figure 2, A).
Adverse events. Freedom from valve reoperation was $97.83 \%$ at 1 year (Figure 2, B). Six patients had valve explant; 3 patients due to endocarditis at 109, 116, and 225 days after implantation, 2 patients due to aortic insufficiency at 111 and 337 days, and 1 at 16 days due to severe valve stenosis caused by a stent deformation after chest compression. Nonstructural valve dysfunction occurred in 10 patients $(3.5 \%)$, endocarditis in 5 patients $(1.7 \%)$, and thromboembolism in 7 patients $(2.4 \%)$. Freedom from stroke was $98.9 \%$ (Figure 2,C). Major bleeding was reported for 38 patients $(13.1 \%)$, of whom $8(2.8 \%)$ required re-exploration. No occurrence of valve thrombosis was seen as confirmed by echocardiographic examinations (Table 3).

Hemodynamics. The overall mean gradient at discharge was $11.8 \mathrm{~mm} \mathrm{Hg}$ and decreased to $9.6 \mathrm{~mm} \mathrm{Hg}$ at 1 year (Table 4). Left ventricle (LV) mass index decreased from $103.5 \pm 30.1 \mathrm{~g} / \mathrm{m}^{2}$ at discharge to $95.8 \pm 27.1 \mathrm{~g} / \mathrm{m}^{2}$ at 1-year follow-up $(P=.001)$. Moderate or greater paravalvular insufficiency was observed in 1 patient $(0.4 \%)$ at discharge and 3 patients $(1.3 \%)$ at 12 -months follow-up. Moderate or greater valvular insufficiency occurred in $0.7 \%(2 / 274)$ patients at discharge and $2.9 \%(7 / 236)$ patients at 1 year (Table E2). Hemodynamic data by valve size are provided in Tables E3 and E4.

Functional health status. Patients' health status improved during follow-up, and $81.2 \%$ of patients increased at least 1 New York Heart Association (NYHA) class. At 1-year follow-up, $97.6 \%$ of patients were in NYHA class I/II (Figure 3). The KCCQ summary score improved from $62.7 \pm 21.8$ before surgery to $85.5 \pm 17.8$ at 1 year $(P<.001)$.

BAV patients. Perceval valve implantation was attempted in 20 patients presenting with congenital BAV and successfully implanted in $17(85 \%)$. Preoperative and operative patient data are shown in Tables 1 and 2. The mean age of the BAV patients was $67.9 \pm 7.1$, with preoperative STS-PROM of $2.0 \pm 1.5$. No deaths, no primary PVL, thromboembolism, or stroke were reported in this group of patients. Two explants occurred due to endocarditis (109 and 225 days after surgery), and 1 new PMI was reported. Hemodynamic findings in the BAV group showed stable improvement in gradients during follow-up. NYHA functional class also improved in $66.7 \%$ of the BAV group at 1-year follow-up, with $93.3 \%$ of patients in NHYA class I/II.

\section{DISCUSSION}

The Perceval valve has been used for AVR since 2007 in multiple European centers participating in consecutive clinical trials. ${ }^{6-10}$ Perceval S valve clinical trial for extended CE mark (CAVALIER) trial initiated in 2010 was the largest, prospective study, involving 658 patients up to a 5 -year follow-up. ${ }^{8-10}$ The present IDE trial details the outcomes 
TABLE 3. Postoperative complications

\begin{tabular}{|c|c|c|c|c|c|c|}
\hline \multirow[b]{2}{*}{ Complications } & \multicolumn{2}{|c|}{ Early (discharge or $\leq \mathbf{3 0}$ d) } & \multicolumn{2}{|c|}{ Total at $6 \mathrm{mo}(0-180 \mathrm{~d})$} & \multicolumn{2}{|c|}{ Total at 1 y $(0-365 \mathrm{~d})$} \\
\hline & $\begin{array}{c}\text { All } \\
(\mathbf{N}=\mathbf{3 0 0}) \mathbf{n}(\%)\end{array}$ & $\begin{array}{c}\text { BAV } \\
(\mathbf{N}=\mathbf{2 0}) \mathbf{n}(\%)\end{array}$ & $\begin{array}{c}\text { All } \\
(N=289) \text { n (\%) }\end{array}$ & $\begin{array}{c}\text { BAV } \\
(\mathbf{N}=17) \text { n }(\%)\end{array}$ & $\begin{array}{c}\text { All } \\
(\mathbf{N}=\mathbf{2 8 9}) \mathbf{n}(\%)\end{array}$ & $\begin{array}{c}\text { BAV } \\
(\mathbf{N}=17) \mathbf{n}(\%)\end{array}$ \\
\hline All deaths & $4(1.3)$ & $0(0)$ & $11(3.8)$ & $0(0)$ & $15(5.2)$ & $0(0)$ \\
\hline Cardiovascular & $3(1.0)$ & $0(0)$ & $7(2.4)$ & $0(0)$ & $9(3.1)$ & $0(0)$ \\
\hline Noncardiovascular & $1(0.3)$ & $0(0)$ & $4(1.4)$ & $0(0)$ & $6(2.1)$ & $0(0)$ \\
\hline Device explant & $1(0.3)$ & $0(0)$ & $2(0.7)$ & $0(0)$ & $6(2.1)$ & $2(11.8)$ \\
\hline Thromboembolism & $5(1.7)$ & $0(0)$ & $6(2.1)$ & $0(0)$ & $7(2.4)$ & $0(0)$ \\
\hline Stroke & $2(0.7)$ & $0(0)$ & $3(1.0)$ & $0(0)$ & $3(1.0)$ & $0(0)$ \\
\hline NSVD & $7(2.3)$ & $0(0)$ & $9(3.1)$ & $0(0)$ & $10(3.5)$ & $0(0)$ \\
\hline Endocarditis & $0(0)$ & $0(0)$ & $4(1.4)$ & $1(5.9)$ & $5(1.7)$ & $2(11.8)$ \\
\hline Hemolysis (minor) & $5(1.7)$ & $0(0)$ & $12(4.1)$ & $1(5.9)$ & $13(4.5)$ & $1(5.9)$ \\
\hline Valve thrombosis & $0(0)$ & $0(0)$ & $0(0)$ & $0(0)$ & $0(0)$ & $0(0)$ \\
\hline New PMI* & $30(10.7)$ & $1(5.0)$ & 35 (12.9) & $1(5.9)$ & $37(13.7)$ & $1(5.9)$ \\
\hline $\begin{array}{l}\text { Atrioventricular block III } \\
\text { leading to new PMI* }\end{array}$ & $7(2.5)$ & $0(0)$ & $8(3.0)$ & $0(0)$ & $8(3.0)$ & $0(0)$ \\
\hline Renal failure & $9(3.0)$ & $1(5.0)$ & $12(4.1)$ & $1(5.9)$ & $15(5.2)$ & $2(11.8)$ \\
\hline Myocardial infarction & $1(0.3)$ & $0(0)$ & $5(1.7)$ & $0(0)$ & $6(2.1)$ & $1(5.9)$ \\
\hline Major bleeding & $32(10.7)$ & $1(5.0)$ & $35(12.1)$ & $1(5.9)$ & $38(13.1)$ & $1(5.9)$ \\
\hline
\end{tabular}

$B A V$, Bicuspid aortic valve; $N S V D$, nonstructural valve dysfunction; $P M I$, pacemaker insertion. *Patients without preoperative pacemaker, $\mathrm{N}=281$ at discharge or $\geq 30$ days, $\mathrm{N}=271$ at 6 months and 1 year.

of the Perceval valve in a multicenter prospective study and represents the initial US experience with the valve. This study was performed with data adjudicated by a Clinical Events Committee, oversight by an independent Data Safety Monitoring Board, and review of echocardiographic studies by a core imaging laboratory.

Perceval was successfully implanted in more than $96 \%$ of patients. The main anatomic reasons for implant exclusion was the presence of aneurysm or enlargement of the ascending aorta with a sino-tubular junction/aortic annulus ratio greater than 1.3. Eleven patients had an unsuccessful implant due to intraoperative PVL, annular size outside the available valve sizes, and aortic root bleeding in 3 patients. The 30-day and 12-month all-cause mortality rates were $1.3 \%$ and $5.2 \%$, respectively, whereas event rates including stroke, endocarditis, device explant, and structural valve dysfunction were low. The Perceval valve generally demonstrated good hemodynamic performance as measured with low transvalvular mean and peak gradients, and regression of LV mass index. Clinical status of patients improved, with $97.6 \%$ of patients enjoying NYHA function class I/II status at 1 year. Furthermore, HR-QoL scores significantly increased from $62.7 \pm 21.8$ before surgery to $85.5 \pm 17.8$ at 1 year $(P<.001)$.

The short crossclamp time and cardiopulmonary bypass time in isolated AVR reported in this series may have potentially contributed to improved outcomes as demonstrated by the low operative mortality compared with the STS-PROM of $2.8 \%$. Similar reductions in operative times corroborate results in the existing literature; the CAVALIER investigators reported crossclamp and cardiopulmonary bypass times of 41 and 65 minutes, respectively. ${ }^{9}$

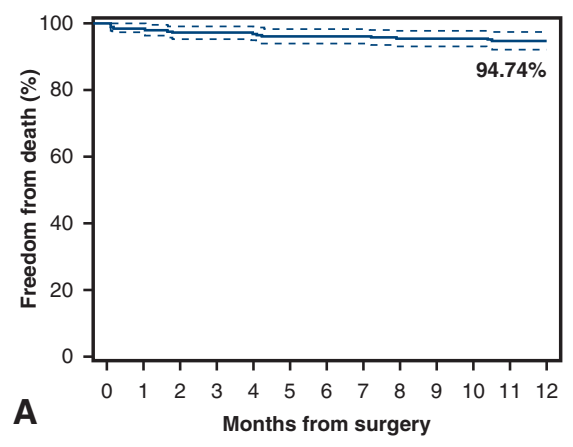

300285281280275273271270266266264258211

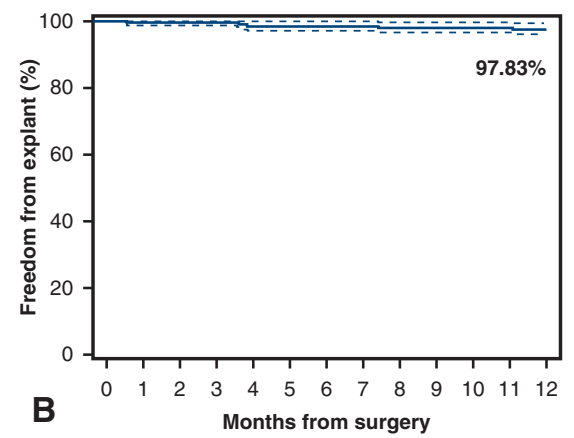

300285281280275273271270266266264258211

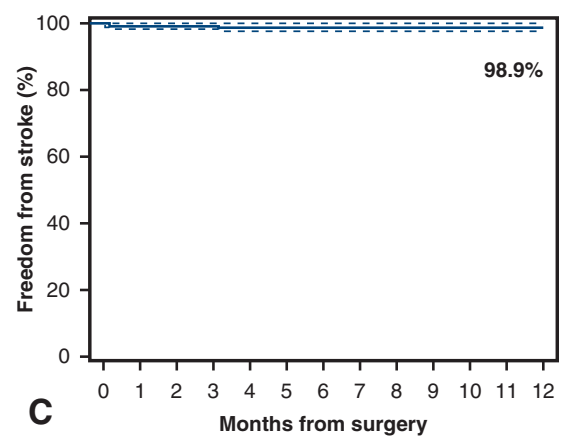

300283279279273271269268264264262256209

FIGURE 2. Survival at 12 months (A), freedom from explants (B), and freedom from stroke (C). 
TABLE 4. Time-related hemodynamic changes

\begin{tabular}{|c|c|c|c|c|c|c|c|c|c|c|c|c|}
\hline & \multicolumn{4}{|c|}{ Discharge/30 d } & \multicolumn{4}{|c|}{ 3-6 mo } & \multicolumn{4}{|c|}{$12 \mathrm{mo}$} \\
\hline & $\mathbf{N}$ & $\begin{array}{c}\text { All, } \\
\text { mean }(\mathrm{SD})\end{array}$ & $\mathbf{N}$ & $\begin{array}{c}\mathrm{BAV}, \\
\text { mean }(\mathrm{SD})\end{array}$ & $\mathbf{N}$ & $\begin{array}{c}\text { All, } \\
\text { mean }(\text { SD) }\end{array}$ & $\mathbf{N}$ & $\begin{array}{c}\text { BAV, } \\
\text { mean }(\mathrm{SD})\end{array}$ & $\mathbf{N}$ & $\begin{array}{c}\text { All, } \\
\text { mean }(\text { SD) }\end{array}$ & $\mathbf{N}$ & $\begin{array}{c}\text { BAV, } \\
\text { mean }(\mathrm{SD})\end{array}$ \\
\hline Aortic mean gradient, $\mathrm{mm} \mathrm{Hg}$ & 245 & $11.8(5.4)$ & 15 & $12.2(4.4)$ & 233 & $10.0(4.9)$ & 15 & $10.9(6.2)$ & 216 & $9.6(4.5)$ & 13 & $10.3(3.7)$ \\
\hline Cardiac output, L/min & 229 & $4.8(1.2)$ & 14 & $5.0(1.1)$ & 233 & $4.8(1.1)$ & 15 & $5.6(1.2)$ & 212 & $4.8(1.1)$ & 13 & $4.9(0.8)$ \\
\hline EOA (TVI), cm & 229 & $1.4(0.4)$ & 14 & $1.4(0.3)$ & 230 & $1.5(0.4)$ & 15 & $1.6(0.5)$ & 213 & $1.5(0.4)$ & 13 & $1.6(0.3)$ \\
\hline EOAi, $\mathrm{cm}^{2} / \mathrm{m}^{2}$ & 229 & $0.7(0.2)$ & 14 & $0.7(0.2)$ & 230 & $0.8(0.2)$ & 15 & $0.8(0.3)$ & 213 & $0.8(0.2)$ & 13 & $0.8(0.2)$ \\
\hline Stroke volume, $\mathrm{mL}$ & 230 & $64.4(15.7)$ & 14 & $66.6(10.7)$ & 233 & $73.7(15.9)$ & 15 & $83.3(15.8)$ & 213 & $75.4(18.1)$ & 13 & $78.3(15.5)$ \\
\hline LV mass, $\mathrm{g}$ & 245 & $200.9(64.5)$ & 14 & $192.3(37.7)$ & 233 & $195.7(63.4)$ & 14 & $182.6(40.8)$ & 220 & $187.5(60.6)$ & 13 & $183.2(35.7)$ \\
\hline LV mass index, $\mathrm{g} / \mathrm{m}^{2}$ & 245 & $103.5(30.1)$ & 14 & $99.1(21.1)$ & 233 & $99.8(28.5)$ & 14 & $93.1(19.2)$ & 220 & $95.8(27.1)$ & 13 & $92.5(20.1)$ \\
\hline
\end{tabular}

$S D$, Standard deviation; $B A V$, bicuspid aortic valve; $E O A$, effective orifice area; $T V I$, time velocity integral; $E O A i$, effective orifice area index; $L V$, left ventricle.

\section{Mortality and Morbidity}

The overall 1-year mortality results in the present study $(94.7 \%)$ are similar to those for the recently published CAVALIER trial $(95 \%))^{9,10}$ Compared with the CAVALIER trial, 30-day mortality was lower in the Perceval US IDE trial (US IDE $1.3 \%$ vs CAVALIER $3.7 \%$ ), as was the mean length of hospital stay (US IDE 8 vs CAVALIER 12 days). This may in part be related to the lower risk profile of patients in the present study (median STS-PROM US IDE 2.8 vs CAVALIER 4.1). Likewise, 12 months after implantation, all-cause mortality $(5.2 \%$ vs $8.1 \%)$, thromboembolism $(2.4 \%$ vs $6.2 \%)$, and stroke rate $(1 \%$ vs $3 \%)$ were lower for in the US IDE trial versus CAVALIER respectively. Recent results from the German Aortic Valve Registry reported greater stroke rates for sutureless and rapid deployment valves as a class compared with standard surgical AVR; however, the rates reported for sutureless/rapid deployment valves were

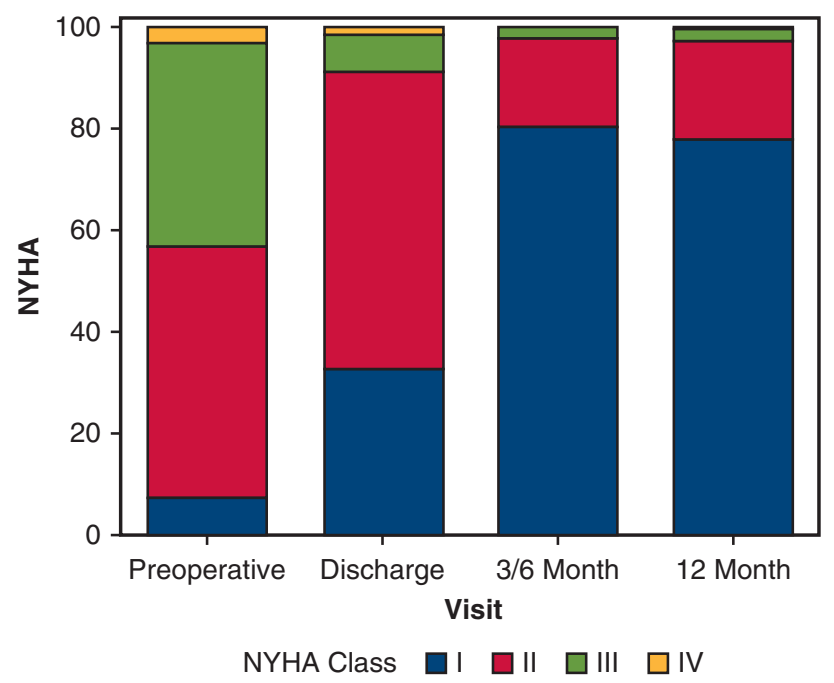

FIGURE 3. NYHA class changes from preoperative to 12 months. NYHA, New York Heart Association. substantially greater than the stroke rate observed in the current study. ${ }^{17}$

Nonstructural valve dysfunction was reported in 10 patients, 7 of whom presented in the early postoperative period and were most likely due to initial malpositioning or oversizing of the valve. The rates of other valve-related adverse events were similarly infrequent and the clinical status of patients significantly improved in both the US IDE and the CAVALIER trials. ${ }^{9,10}$

Although a significant majority of patients were successfully implanted, early experience with a novel technology likely contributed in some cases. There is no clear explanation for a somewhat-elevated occurrence of major bleeding compared with what might be expected for standard AVR; however, this complication has not previously been reported as a significant issue in the literature for the Perceval valve. In 3 cases, implantation was unsuccessful due to significant root complications (annular bleeding in 2 cases; limited aortic dissection requiring root replacement in one case). Aortic root bleeding observed in this study was likely the result of annular debridement resulting in a full-thickness injury to the aortic annulus. Suture placement during standard surgical AVR might normally be expected to repair this type of injury during implant. It is important to note that due to the absence of annular sutures, full-thickness disruptions of the aortic wall should be identified and securely repaired before Perceval implantation.

In the present study, the need for new PMI appears to be greater compared with conventional valves, with the reported need for PMI ranging from $3.0 \%$ to $11.8 \%$ after conventional AVR. ${ }^{18}$ The Perceval IDE trial represents the initial US experience for the Perceval valve, and there has been a subsequent emphasis on approaches to avoid damage to the conduction system during implantation with particular attention to the depth of implantation. Current practice has evolved with demonstration that optimal positioning of the valve is achieved by placement of the guiding sutures, approximately 2 to $3 \mathrm{~mm}$ below the leaflet hinge point as 
stated in the current US Instructions for Use to avoid damage to the conduction system, while maintaining secure placement of the valve.

The importance of implantation depth for pacemaker implantation is highlighted by the recent report from Yanagawa and colleagues, ${ }^{19}$ who identified an association between deeper positioning of the prosthesis in the LV outflow tract and postoperative conduction disorders. After modifying their implantation technique by placing the guiding sutures closer to the point of leaflet insertion in the annulus, the authors reduced their pacemaker implantation rate for Perceval from $28 \%$ to $0 \%$. Fischlein and colleagues $^{20}$ also reported improvement for pacemaker rates from an initial value of $10 \%$ to a current rate of $3 \%$, as they achieved greater experience with this new technology. Recently published large registry experiences ${ }^{17,21}$ have also reported greater PMI rates compared with standard surgical AVR; however, results from Sutureless and Rapid-Deployment Aortic Valve Replacement International Registry, an international registry of more than 3300 patients undergoing sutureless or rapid deployment AVR, reported a significant decrease in PMI rates over time that the authors attributed to the learning curve for these new valve technologies (from $17.2 \%$ in 2007 to $5.4 \%$ in 2016).

\section{Hemodynamics}

Our study corroborates previous European trials that have demonstrated good hemodynamic performance of Perceval. Significant reduction of transvalvular peak and mean pressure gradient in addition to increased EOA index of the valve resulted in significant regression of LV mass, accompanied by improved NYHA classification status and HR-QoL scores. Overall discharge transprosthesis mean gradients were minimally greater than the CAVALIER trial (11.8 $\mathrm{mm} \mathrm{Hg}$ vs $10.2 \mathrm{~mm} \mathrm{Hg}$ ), whereas EOA values were comparable with CAVALIER results. ${ }^{10}$ Mean gradients continued to decrease after discharge and were $9.6 \mathrm{~mm}$ $\mathrm{Hg}$ for the overall cohort at 1 year of follow-up.

Small sizes showed a greater rate of PPM along with greater transvalvular gradients that decreased at 1-year follow-up. Recent literature has demonstrated that PPM after standard AVR in small-sized bioprostheses is a common finding, particularly when rigorous core laboratory assessments are performed. ${ }^{22}$ PPM is also greatly affected by patient selection, and the implanting surgeon must consider the appropriate valve and implant techniques required to minimize high gradients and resultant PPM. Rates of moderate or greater isolated PVL were around $1 \%$ in the present study, and the presence of moderate or greater central aortic regurgitation was less than $3 \%$ at 1-year follow-up. PERSIST-AVR (Perceval Sutureless Implant Versus Standard Aortic Valve Replacement trial, NCT02673697) is an ongoing randomized control trial comparing Perceval to standard surgical AVR and will be instructive to further establish hemodynamic outcomes.

\section{Functional Status and Quality of Life}

Consistent with the reported postoperative hemodynamics, significant and stable improvement of NYHA functional class was observed in the majority of patients. More than $80 \%$ of patients in the current trial demonstrated functional status improvement, and $97 \%$ of patients were in NYHA class I or II at 1 year. HR-QoL, as measured by the KCCQ summary score, also improved significantly at 12 months, demonstrating a substantial clinical improvement in this patient group. Isolated AVR patients from the Perceval IDE study had a mean increase of more than 20 points in the HR-QOL score at follow-up (22 from baseline to 3-6 months; 22.3 from baseline to 12 months).

\section{BAV Patients}

Although a minority of patients in this trial had BAV morphology $(\mathrm{N}=20)$, the present study represents a unique experience compared with previous European trials, which excluded this population. Several recent publications have addressed BAV patients and their potential suitability for Perceval valve implantation. An International Expert Consensus Panel publication advised that congenital BAV patients without raphe (Sievers Type 0) should be avoided for sutureless/rapid deployment AVR, whereas those with 1 or 2 raphes (Sievers Type 1 and 2, respectively) might be considered contingent upon appropriate dimensions of the aortic annulus and ascending aorta. ${ }^{23}$ Clinical series recently reported the outcomes of BAV patients successfully managed with Perceval and have provided guidance regarding patient selection and surgical technique. ${ }^{24,25}$ The results of the current report confirm that appropriately selected BAV patients can be successfully treated with Perceval valve.

\section{Limitations}

One of the limitations of this study is the relatively short period of follow-up (1 year). These short-term results will need to be supported by studies of greater duration to gain a complete understanding of long-term outcomes for Perceval.

The relatively small proportion of BAV patients included in the present study might be due to the frequent association of this condition with aortic root enlargement or aneurysm, which were exclusion criteria in this trial, therefore potentially reducing the number of eligible BAV patients. Another limitation is incomplete clinical data arising from 11 unsuccessfully implanted patients. These patients were withdrawn from the study and as per study protocol, their clinical data after the perioperative period were not collected. This is a prospective single-arm study that was not intended as a comparative study against other valves, 
and so is unable to demonstrate superiority or noninferiority in terms of safety and effectiveness versus any other technology. Larger randomized trials are required to provide Level I evidence in this regard. As mentioned, a large prospective, randomized controlled trial comparing the Perceval valve with standard surgical AVR is currently ongoing to provide greater understanding of the role of Perceval in the surgical management of aortic valve disease. Finally, since the Perceval IDE study is an investigational study of selected patients meeting specific inclusion/exclusion criteria, more data from real-world experience will be needed to fully understand the performance in the general patient population further confirm the findings from this study.

\section{CONCLUSIONS}

These US IDE trial results confirm the safety and efficacy of the Perceval sutureless bioprosthesis for replacement of the diseased aortic valve in selected study patients. Despite implantation failure in 3 patients $(3 / 300)$ due to aortic annular bleeding, we observed low 1-year mortality, stroke, explantation, and valve-related event rates along with stable hemodynamic performance that accompanied significant reduction of transvalvular pressure gradients and LV mass index. Patient quality of life improved significantly, and greater than $90 \%$ of the cohort were in NYHA class of I/II after 1 year of follow-up. PMI and aortic regurgitation are inherent potential risks with the Perceval valve, and randomized trials comparing the Perceval to standard sutured prostheses will be instructive to further define outcomes.

\section{Conflict of Interest Statement}

Dr Heimansohn: Proctor LivaNova; Patient Selection Committee, Abbott Vascular. Dr Weissmann: research support recipient from Boston Scientific, Medtronic, Edwards Lifesciences, St. Jude Medical, Abbott Vascular, LivaNova. Dr Ailawadi: speaker for Atricure, Convatec; consultant for Abbott Vascular, Edwards Lifesciences, Medtronic, St Jude Medical. Dr Ad: consultant for Cryolife, Atricure, Medtronic, LivaNova; shareholder of Nido Surgical; coowner LAA occlusion LLC. Dr Thourani: consultant for Edwards Lifesciences, Abbott Vascular; research recipient from Edwards Lifesciences, Abbott Vascular, Boston Scientific, Medtronic. Dr Szeto: speaker for Abbott Vascular, St Jude Medical; consultant for Microinterventional Device; research support recipient from LivaNova, Edwards Lifesciences, Medtronic. Dr Michler: NIH U01 Principal Investigator; Dr Michelena: advisor of Cryolife On-X valve. Dr Dabir: proctor for LivaNova. Dr Moront: Medtronic Heart Valves, consultant and proctor; Edwards LifeSciences, consultant; LSI, consultant; Terumo, consult and trainer. Dr Subramanian: consultant and proctor for LivaNova. Dr Roselli: Speaker for Edwards Lifesciences, St Jude Medical, Vascutek; consultant for Medtronic, LivaNova. All other authors have nothing to disclose with regard to commercial support

The authors are grateful to LivaNova employees Brian Duncan, MD, Giovanni Goisis, MStat, Mara Chiaro, DVM, and Sara Gaggianesi, DVM, for technical support in the analysis of data and manuscript preparation.

\section{References}

1. Nishimura RA, Otto CM, Bonow RO, Carabello BA, Erwin JP, Guyton RA, et al. 2014 AHA/ACC guideline for the management of patients with valvular heart disease: a report of the American College of Cardiology/American Heart Association task force on practice guidelines. J Am Coll Cardiol. 2014;63:e57-185.

2. Wilbring M, Alexiou K, Schumann E, Matschke K, Tugtekin SM. Isolated aortic valve replacement in patients with small aortic annulus - a high-risk group on long-term follow-up. Thorac Cardiovasc Surg. 2013;61:379-85.

3. Antunes MJ. Editorial comment: aortic stenosis in octogenarians and other highrisk groups: what can surgical valve replacement offer? Eur J Cardiothorac Surg. 2012;42:940-1.

4. Santarpino G, Pfeiffer S, Fischlein T. Favourable outcomes after high-risk conventional aortic valve replacement: can we do even better? Eur J Cardiothorac Surg. 2012;41:1218-9.

5. Concistre G, Farneti P, Miceli A, Glauber M. Sutureless aortic bioprosthesis in severe aortic root calcification: an innovative approach. Interact Cardiovasc Thorac Surg. 2012;14:670-2.

6. Meuris B, Flameng WJ, Laborde F, Folliguet TA, Haverich A, Shrestha M. Fiveyear results of the pilot trial of a sutureless valve. J Thorac Cardiovasc Surg. 2015;150:84-8.

7. Shrestha M, Folliguet TA, Pfeiffer S, Meuris B, Carrel T, Bechtel M, et al. Aortic valve replacement and concomitant procedures with the Perceval valve: results of European trials. Ann Thorac Surg. 2014;98:1294-300.

8. Shrestha M, Fischlein T, Meuris B, Flameng W, Carrel T, Madonna F, et al. European multicentre experience with the sutureless Perceval valve: clinical and haemodynamic outcomes up to 5 years in over 700 patients. Eur J Cardiothorac Surg. 2016;49:234-41.

9. Laborde F, Fischlein T, Hakim-Meibodi K, Misfeld M, Carrel T, Zembala M, et al. Clinical and haemodynamic outcomes in 658 patients receiving the Perceval sutureless aortic valve: early results from a prospective European multicentre study (the Cavalier Trial). Eur J Cardiothorac Surg. 2016;49:978-86.

10. Fischlein T, Meuris B, Hakim-Meibodi K, Misfeld M, Carrel T, Zembala M, et al The sutureless aortic valve at 1 year: a large multicenter cohort study. J Thorac Cardiovasc Surg. 2016;151:1617-26.E4.

11. Mazine A, Teoh K, Bouhout I, Bhatnagar G, Pelletier M, Voisine P, et al. Sutureless aortic valve replacement: a Canadian multicentre study. Can J Cardiol. 2015; $31: 63-8$.

12. Shrestha M, Folliguet T, Meuris B, Dibie A, Bara C, Herregods M-C, et al Sutureless Perceval S aortic valve replacement: a multicenter, prospective pilot trial. J Heart Valve Dis. 2009;18:698-702.

13. Zoghbi WA, Chambers JB, Dumesnil JG, Foster E, Gottdiener JS, Grayburn PA et al. Recommendations for evaluation of prosthetic valves with echocardiography and Doppler ultrasound: a report from the American Society of Echocardiography's guidelines and standards committee and the task force on prosthetic valves, developed in conjunction with the American College of Cardiology cardiovascular imaging committee, cardiac imaging committee of the American Heart Association, the European Association of Echocardiography, a registered branch of the European Society of Cardiology, the Japanese Society of Echocardiography and the Canadian Society of Echocardiography, endorsed by the American College of Cardiology foundation, American Heart Association, European Association of Echocardiography, a registered branch of the European Society of Cardiology, the Japanese Society of Echocardiography, and Canadian Society of Echocardiography. J Am Soc Echocardiogr. 2009;22:975-1014.

14. Green CP, Porter CB, Bresnahan DR, Spertus JA. Development and evaluation of the Kansas city cardiomyopathy questionnaire: a new health status measure for heart failure. J Am Coll Cardiol. 2000;35:1245-55.

15. Andersen PK, Borgan O, Gill RD, Keiding N. Statistical Models Based on Count ing Processes. New York: Springer Verlag; 1993.

16. Akins CW, Miller DC, Turina MI, Kouchoukos NT, Blackstone EH, Grunkemeier GL, et al. Guidelines for reporting mortality and morbidity after cardiac valve interventions. Ann Thorac Surg. 2008;85:1490-5. 
17. Ensminger S, Fujita B, Bauer T, Möllmann H, Beckmann A, Bekeredjian R, et al; The GARY Executive Board. Rapid deployment versus conventional bioprosthetic valve replacement for aortic stenosis. J Am Coll Cardiol. 2018;71:1417-28.

18. Matthews IG, Fazal IA, Bates MG, Turley AJ. In patients undergoing aortic valve replacement, what factors predict the requirement for permanent pacemaker implantation? Interact Cardiovasc Thorac Surg. 2011;12:475-9.

19. Yanagawa B, Cruz J, Boisvert L, Bonneau D. A simple modification to lower incidence of heart block with sutureless valve implantation. J Thorac Cardiovasc Surg. 2016;152:630-2.

20. Fischlein T, Dell'Aquila AM, Santarpino G. Technical changes in the implant of sutureless aortic valves: the sense of being pioneers. J Thorac Cardiovasc Surg. 2016;152:288.

21. Di Eusanio M, Phan K, Berretta P, Carrel TP, Andreas M, Santarpino G, et al. Sutureless and rapid-deployment aortic valve replacement international registry (SURD-IR): early results from 3343 patients. Eur J Cardiothorac Surg. 2018; 54:768-73.

22. Pibarot P, Weissman NJ, Stewart WJ, Hahn RT, Lindman BR, McAndrew T, et al Incidence and sequelae of prosthesis-patient mismatch in transcatheter versus surgical valve replacement in high-risk patients with severe aortic stenosis: a PARTNER trial cohort-A analysis. J Am Coll Cardiol. 2014;64:1323-34.

23. Gersak B, Fischlein T, Folliguet TA, Meuris B, Teoh KHT, Moten SC, et al. Sutureless, rapid deployment valves and stented bioprosthesis in aortic valve replacement: recommendations of an international expert consensus panel. Eur J Cardiothorac Surg. 2016;49:709-18.

24. Nguyen A, Fortin W, Mazine A, Bouchard D, Carrier M, El Hamamsy I, et al. Sutureless aortic valve replacement in patients who have bicuspid aortic valve. J Thorac Cardiovasc Surg. 2015;150:851-7.

25. Glauber M, Ferrarini M, Lio A, Miceli A. Dealing with a stenotic bicuspid aortic valve: is this still an off-label procedure for a sutureless valve? J Thorac Cardiovasc Surg. 2015;150:858-9.

Key Words: aortic valve replacement, sutureless valve, aortic stenosis, minimally invasive surgery, hemodynamics, quality of life, concomitant procedures 
PERCEVAL IDE

Failure to Implant $(n=11)$

- Paravalvular leak $(n=4)$

- Aortic annular bleeding $(n=2)$

- Device dislodgement $(n=2)$

- Congenital bicuspid $(n=1)$

- Aortic dissection ( $n=1)$

- Annulus outside the study parameters $(n=1)$

Withdrawn/Lost to Follow-up $(n=4)$

- Patient death $(n=4)$

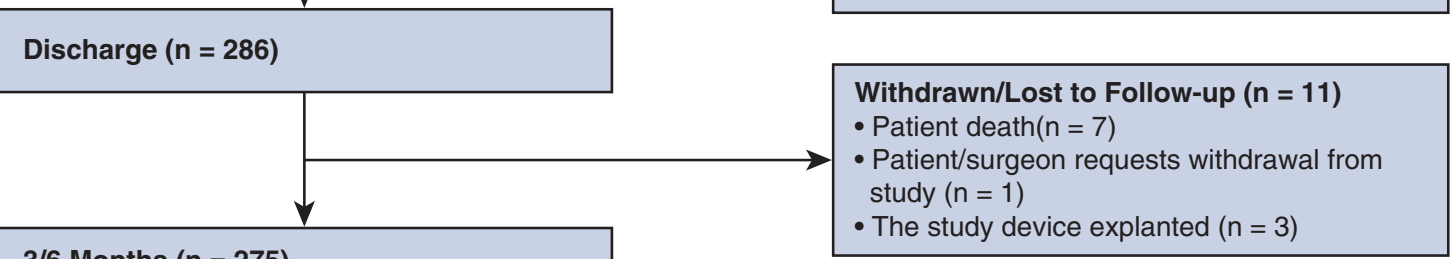

3/6 Months ( $n=275)$

- Patient with assessment $(n=265)$

- Patient without assessment $(n=10)$

12 Months ( $n=259$ )

- Patient with assessment $(n=245)$

- Patient without assessment $(n=14)$

FIGURE E1. Patient flow chart of the Perceval Investigational Device Exemption trial. IDE, Investigational Device Exemption.

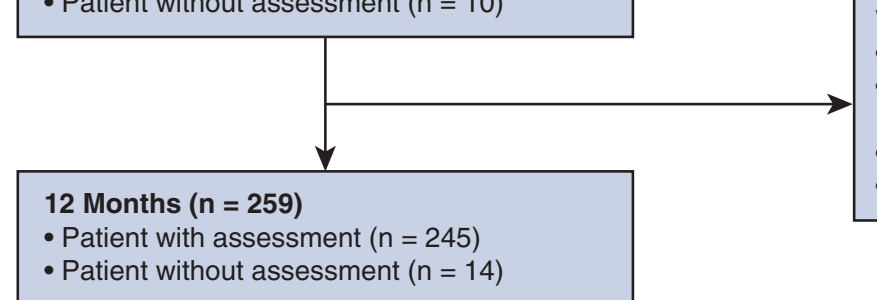

Withdrawn/Lost to Follow-up $(n=16)$

- Patient death $(n=4)$

- Patient/surgeon requests withdrawal from

study $(n=7)$

- The study device explanted $(n=3)$

- Missing $(n=2)$ 
TABLE E1. Inclusion and exclusion criteria

\section{Inclusion criteria}

1. Subjects of age $>18$ years.

2. Subjects with aortic valve stenosis or stenoinsufficiency.

3. The subject is willing to sign the informed consent.

4. The subject in which preoperative evaluation indicated the need for native or prosthetic aortic valve replacement.

5. The subject is located in a geographic location that will enable the subject to return to the study site for all follow-up examinations (ie, geographically stable).

6. Subject will be available to the investigator(s) for postoperative follow-up beyond 1 year.

\section{Exclusion criteria}

1. The subject has pre-existing valve prosthesis or annuloplasty ring in the mitral, pulmonic, or tricuspid position.

2. The subject requires a double or multiple valve replacement or repair of the mitral, tricuspid, or pulmonic valve.

3. The subject has a previously implanted Perceval valve that requires replacement

4. Subjects requiring simultaneous cardiac procedures, apart from septal myectomy and/or coronary bypass.

5. The subject has active endocarditis.

6. Subjects with active myocarditis.

7. The subject is or will be participating in a concomitant research study of an investigational product.

8. Subjects with aneurysmal dilation or dissection of the ascending aortic wall.

9. The subject is a minor, drug abuser, alcohol abuser, prison inmate, institutionalized, or is unable to give informed consent.

10. The subject has a major or progressive noncardiac disease that, in the investigator's experience, results in a life expectancy of less than 1 year, or the implant of the device produces an unacceptable increased risk to the patient.

11. Subjects with known hypersensitivity to nickel alloys.

12. The subject is undergoing renal dialysis for chronic renal failure or has hyperparathyroidism.

13. The subject has had an acute preoperative neurologic deficit, myocardial infarction, or cardiac event that has not returned to baseline or stabilized for $\geq 30$ days before the planned valve implant surgery.

14. Subject is known to be noncompliant or is unlikely to complete the study.

15. Subjects with an aortic root enlargement, where the ratio between the diameter of the sino-tubular junction and the annulus diameter, assessed by TTE, is $>1.3$.

$T T E$, Transthoracic echocardiography

TABLE E2. Aortic regurgitation-echocardiographic core laboratory assessment

\begin{tabular}{|c|c|c|c|c|c|c|c|c|c|c|c|c|}
\hline & \multicolumn{4}{|c|}{ Discharge/30 d } & \multicolumn{4}{|c|}{ 3-6 mo } & \multicolumn{4}{|c|}{$12 \mathrm{mo}$} \\
\hline & All $(N=274)$ & $\%$ & BAV $(\mathbf{N}=17)$ & $\%$ & All $(N=249)$ & $\%$ & BAV $(\mathbf{N}=15)$ & $\%$ & All $(\mathbf{N}=236)$ & $\%$ & BAV $(\mathbf{N}=13)$ & $\%$ \\
\hline \multicolumn{13}{|l|}{ PVL } \\
\hline None/trace & 250 & 91.2 & 12 & 70.6 & 221 & 88.8 & 15 & 100 & 217 & 91.9 & 13 & 100 \\
\hline Mild & 8 & 2.9 & 2 & 11.8 & 13 & 5.2 & 0 & 0 & 9 & 3.8 & 0 & 0 \\
\hline Moderate or greater & 1 & 0.4 & 0 & 0 & 3 & 1.2 & 0 & 0 & 3 & 1.3 & 0 & 0 \\
\hline Not evaluable & 15 & 5.5 & 3 & 17.6 & 12 & 4.8 & 0 & 0 & 7 & 3.0 & 0 & 0 \\
\hline \multicolumn{13}{|l|}{ Central regurgitation } \\
\hline None/trace & 201 & 73.4 & 9 & 52.9 & 163 & 65.5 & 10 & 66.7 & 137 & 58.1 & 7 & 53.8 \\
\hline Mild & 56 & 20.4 & 5 & 29.4 & 65 & 26.1 & 3 & 20.0 & 83 & 35.2 & 4 & 30.8 \\
\hline Moderate or greater & 2 & 0.7 & 0 & 0 & 11 & 4.4 & 2 & 13.3 & 7 & 2.9 & 2 & 15.4 \\
\hline Not evaluable & 15 & 5.5 & 3 & 17.6 & 10 & 4.0 & 0 & 0 & 9 & 3.8 & 0 & 0 \\
\hline \multicolumn{13}{|l|}{ Total aortic regurgitation } \\
\hline None/trace & 197 & 71.9 & 9 & 52.9 & 155 & 62.2 & 10 & 66.7 & 132 & 55.9 & 7 & 53.8 \\
\hline Mild & 65 & 23.7 & 5 & 29.4 & 71 & 28.5 & 3 & 20.0 & 91 & 38.6 & 4 & 30.8 \\
\hline Moderate or greater & 5 & 1.8 & 1 & 5.9 & 20 & 8.0 & 2 & 13.3 & 13 & 5.5 & 2 & 15.4 \\
\hline Not evaluable & 7 & 2.6 & 2 & 11.8 & 3 & 1.2 & 0 & 0 & 0 & 0 & 0 & 0 \\
\hline
\end{tabular}

$B A V$, Bicuspid aortic valve; $P V L$, paravalvular leak 
TABLE E3. Hemodynamic performance evaluated by transthoracic echocardiography

\begin{tabular}{|c|c|c|c|c|c|c|}
\hline & \multicolumn{2}{|c|}{ Discharge/30 d } & \multicolumn{2}{|c|}{ 3-6 mo } & \multicolumn{2}{|c|}{$12 \mathrm{mo}$} \\
\hline & $\mathbf{N}$ & $\begin{array}{l}\text { Mean (SD) } \\
{[\text { min-max] }}\end{array}$ & $\mathbf{N}$ & $\begin{array}{l}\text { Mean (SD) } \\
{[\text { min-max] }}\end{array}$ & $\mathbf{N}$ & $\begin{array}{l}\text { Mean (SD) } \\
{[\text { min-max] }}\end{array}$ \\
\hline \multicolumn{7}{|c|}{ Aortic mean gradient, $\mathrm{mm} \mathrm{Hg}$} \\
\hline Size $S$ & 17 & $16.0(6.9)$ & 15 & $13.5(8.9)$ & 13 & $12.6(5.6)$ \\
\hline Size M & 79 & $13.6(5.9)$ & 72 & $11.5(4.4)$ & 66 & $10.9(4.9)$ \\
\hline Size L & 107 & $11.0(4.7)$ & 103 & $9.4(4.4)$ & 98 & $9.0(3.8)$ \\
\hline Size XL & 42 & $8.8(3.4)$ & 43 & $7.6(2.9)$ & 39 & $7.7(4.1)$ \\
\hline \multicolumn{7}{|c|}{ EOA (TVI), $\mathrm{cm}^{2}$} \\
\hline Size $S$ & 16 & $1.1(0.2)$ & 15 & $1.1(0.2)$ & 13 & $1.1(0.2)$ \\
\hline Size M & 73 & $1.3(0.3)$ & 70 & $1.4(0.4)$ & 66 & $1.4(0.4)$ \\
\hline Size L & 101 & $1.5(0.4)$ & 102 & $1.6(0.4)$ & 95 & $1.6(0.3)$ \\
\hline Size XL & 39 & $1.7(0.4)$ & 43 & $1.7(0.4)$ & 39 & $1.7(0.4)$ \\
\hline \multicolumn{7}{|c|}{ EOAi, $\mathrm{cm}^{2} / \mathrm{m}^{2}$} \\
\hline Size $S$ & 16 & $0.6(0.2)$ & 15 & $0.6(0.2)$ & 13 & $0.7(0.2)$ \\
\hline Size M & 73 & $0.7(0.2)$ & 70 & $0.8(0.2)$ & 66 & $0.8(0.2)$ \\
\hline Size L & 101 & $0.7(0.2)$ & 102 & $0.8(0.2)$ & 95 & $0.8(0.2)$ \\
\hline Size XL & 39 & $0.8(0.2)$ & 43 & $0.8(0.2)$ & 39 & $0.8(0.2)$ \\
\hline \multicolumn{7}{|c|}{ LVOT diameter, $\mathrm{cm}$} \\
\hline \multirow[t]{2}{*}{ Size $S$} & 17 & $1.8(0.1)$ & 18 & $1.8(0.1)$ & 16 & $1.8(0.1)$ \\
\hline & & {$[1.7 ; 2.0]$} & & {$[1.7 ; 1.9]$} & & {$[1.7 ; 1.9]$} \\
\hline \multirow[t]{2}{*}{ Size M } & 82 & $1.9(0.1)$ & 75 & $1.9(0.1)$ & 73 & $1.9(0.1)$ \\
\hline & & {$[1.7 ; 2.2]$} & & {$[1.7 ; 2.2]$} & & {$[1.6 ; 2.2]$} \\
\hline \multirow[t]{2}{*}{ Size L } & 115 & $2.0(0.1)$ & 110 & $2.0(0.1)$ & 101 & $2.0(0.1)$ \\
\hline & & {$[1.8 ; 2.4]$} & & {$[1.8 ; 2.4]$} & & {$[1.7 ; 2.4]$} \\
\hline \multirow[t]{2}{*}{ Size XL } & 45 & $2.1(0.1)$ & 44 & $2.1(0.1)$ & 42 & $2.1(0.1)$ \\
\hline & & {$[1.9 ; 2.3]$} & & {$[1.8 ; 2.4]$} & & {$[1.8 ; 2.4]$} \\
\hline \multicolumn{7}{|l|}{ LV mass, $\mathrm{g}$} \\
\hline Size $S$ & 18 & $164.6(75.9)$ & 18 & $163.7(63.3)$ & 16 & $166.5(57.7)$ \\
\hline Size M & 80 & $182.3(56.1)$ & 75 & $178.3(59.5)$ & 71 & $171.0(54.4)$ \\
\hline Size L & 106 & $200.9(52.9)$ & 101 & $199.4(56.5)$ & 93 & $186.6(56.2)$ \\
\hline Size XL & 41 & $253.2(72.6)$ & 39 & $234.1(69.8)$ & 40 & $227.3(65.2)$ \\
\hline \multicolumn{7}{|c|}{ LV mass index, $\mathrm{g} / \mathrm{m}^{2}$} \\
\hline Size S & 18 & $91.8(35.8)$ & 18 & $91.8(30.1)$ & 16 & $94.0(28.5)$ \\
\hline Size M & 80 & $99.4(25.9)$ & 75 & $96.4(27.4)$ & 71 & $93.1(26.1)$ \\
\hline Size L & 106 & $100.8(27.5)$ & 101 & $98.5(26.0)$ & 93 & $91.7(24.8)$ \\
\hline Size XL & 41 & $123.6(34.0)$ & 39 & $113.8(32.5)$ & 40 & $110.5(29.3)$ \\
\hline
\end{tabular}

TABLE E4. Patient prosthesis mismatch $($ EOAi $<0.65)$

\begin{tabular}{|c|c|c|c|c|c|c|c|c|c|c|}
\hline \multirow[b]{2}{*}{ Follow-up } & \multirow{2}{*}{\multicolumn{2}{|c|}{ Overall }} & \multicolumn{8}{|c|}{ Valve size } \\
\hline & & & \multicolumn{2}{|r|}{$\mathbf{S}$} & \multicolumn{2}{|r|}{$\mathbf{M}$} & \multicolumn{2}{|r|}{$\mathbf{L}$} & \multicolumn{2}{|r|}{$\mathbf{X L}$} \\
\hline \multirow[t]{2}{*}{ Discharge } & $\mathrm{N}$ & $\%(\mathrm{~N}=283)$ & $\mathrm{N}$ & $\%(\mathrm{~N}=19)$ & $\mathrm{N}$ & $\%(\mathrm{~N}=93)$ & $\mathrm{N}$ & $\%(\mathrm{~N}=123)$ & $\mathrm{N}$ & $\%(\mathrm{~N}=48)$ \\
\hline & 91 & $32.2 \%$ & 8 & $42.1 \%$ & 30 & $32.3 \%$ & 41 & $33.3 \%$ & 12 & $25.0 \%$ \\
\hline \multirow[t]{2}{*}{ 3-6 mo } & $\mathrm{N}$ & $\%(\mathrm{~N}=256)$ & $\mathrm{N}$ & $\%(\mathrm{~N}=18)$ & $\mathrm{N}$ & $\%(\mathrm{~N}=80)$ & $\mathrm{N}$ & $\%(\mathrm{~N}=114)$ & $\mathrm{N}$ & $\%(\mathrm{~N}=44)$ \\
\hline & 79 & $30.9 \%$ & 11 & $61.1 \%$ & 28 & $35.0 \%$ & 34 & $29.8 \%$ & 6 & $13.6 \%$ \\
\hline \multirow[t]{2}{*}{$12 \mathrm{mo}$} & $\mathrm{N}$ & $\%(\mathrm{~N}=243)$ & $\mathrm{N}$ & $\%(\mathrm{~N}=16)$ & $\mathrm{N}$ & $\%(\mathrm{~N}=77)$ & $\mathrm{N}$ & $\%(\mathrm{~N}=106)$ & $\mathrm{N}$ & $\%(\mathrm{~N}=44)$ \\
\hline & 68 & $28.0 \%$ & 6 & $37.5 \%$ & 25 & $32.5 \%$ & 27 & $25.5 \%$ & 10 & $22.7 \%$ \\
\hline
\end{tabular}

EOA $i$, Effective orifice area index. 